JOURNAL OF

FUNCTION SPACES AND APPLICATIONS

Volume 7, Number 3 (2009), 289-300
(C) 2009, Scientific Horizon http://www.jfsa.net

\title{
On Bellman-Golubov theorems for the Riemann-Liouville operators
}

\author{
Pham Tien Zung \\ (Communicated by Victor Burenkov)
}

2000 Mathematics Subject Classification. Primary 26D10; Secondary 26D15, $26 \mathrm{D} 07$.

Keywords and phrases. Fourier integral, Lebesgue spaces, Riemann-Liouville operator.

Abstract. Superposition of Fourier transform with the Riemann - Liouville operators is studied.

\section{Introduction}

Let $\mathbb{R}:=(-\infty,+\infty)$. We denote $\|f\|_{p}:=\left(\int_{\mathbb{R}}|f(x)|^{p} d x\right)^{1 / p}$ for $1 \leq p<$ $\infty$ and $\|f\|_{\infty}:=\operatorname{esssup}_{x \in \mathbb{R}}|f(x)|$. By $L^{p}(\mathbb{R})$ we denote the Lebesgue space of all measurable functions on $\mathbb{R}$ such that $\|f\|_{p}<\infty$. Similar notations are applied for $\mathbb{R}_{+}:=[0,+\infty)$.

For $f \in L^{1}(\mathbb{R})$, the Fourier transform $F f$ is defined by

$$
F f(x):=\frac{1}{\sqrt{2 \pi}} \int_{-\infty}^{\infty} f(t) e^{i x t} d t
$$

In particular cases, when $f$ is even or odd, the Fourier transforms are

$$
F_{c} f(x):=\sqrt{\frac{2}{\pi}} \int_{0}^{\infty} f(t) \cos x t d t
$$


and

$$
i F_{s} f(x):=i \sqrt{\frac{2}{\pi}} \int_{0}^{\infty} f(t) \sin x t d t
$$

respectively. $F_{c} f$ and $F_{s} f$ are called the cosine and sine Fourier transforms and may be independently defined for a function on $\mathbb{R}_{+}$. It is well known $\left[6\right.$, Theorem 74] that for $f \in L^{p}(\mathbb{R}), 1<p \leq 2$, there exist $F f \in L^{p^{\prime}}(\mathbb{R})$ such that $\lim _{a \rightarrow \infty}\left\|F f-F f_{a}\right\|_{p^{\prime}}=0$, where $p^{\prime}=\frac{p}{p-1}$ and

$$
F f_{a}(x):=\frac{1}{\sqrt{2 \pi}} \int_{-a}^{a} e^{i x t} f(t) d t, a>0 .
$$

Moreover, the Planscherel-Titchmarsh inequality

$$
\|F f\|_{p^{\prime}} \leq C(p)\|f\|_{p}
$$

holds. The similar results are valid for the sine and cosine Fourier transforms.

R. Bellman [1] stated and B. I. Golubov [3] proved the following equalities:

$$
P F_{c} f=F_{c} Q f
$$

if $f \in L^{p}(\mathbb{R}), 1 \leq p \leq 2$ and

$$
Q F_{c} f=F_{c} P f
$$

if $f \in L^{p}(\mathbb{R}), 1<p \leq 2$, where

$$
P f(x):=\frac{1}{x} \int_{0}^{x} f(s) d s
$$

and

$$
Q f(x):=\int_{x}^{\infty} \frac{f(s)}{s} d s
$$

are the Hardy operators.

The aim of the paper is to prove the equalities similar to (2) and (3), where the Hardy operators $P$ and $Q$ are replaced by the Riemann-Liouville operators.

By $C$, we denote constants, which may be different in different occurences. 


\section{Main results}

Let $\alpha>0$. The Riemann-Liouville operators are defined for a function on the semiaxis $\mathbb{R}_{+}$as follows:

$$
B_{\alpha} f(x):=\frac{1}{x^{\alpha}} \int_{0}^{x}(x-t)^{\alpha-1} f(t) d t
$$

and

$$
H_{\alpha} f(x):=\int_{x}^{\infty} \frac{(t-x)^{\alpha-1}}{t^{\alpha}} f(t) d t .
$$

It is known [4, Theorem 329] that

$$
\left\|B_{\alpha} f\right\|_{p} \leq C_{\alpha, p}\|f\|_{p}
$$

for $1<p \leq \infty$ and

$$
\left\|H_{\alpha} f\right\|_{p} \leq C_{\alpha, p}\|f\|_{p}
$$

for $1 \leq p<\infty$.

Theorem 1. Let $1<p \leq 2$ and $\alpha>1 / p^{\prime}$ and suppose that $f \in L^{p}\left(\mathbb{R}_{+}\right)$. Then

$$
B_{\alpha}\left[F_{c} f\right](x)=F_{c}\left[H_{\alpha} f\right](x), \quad \text { a.e } x \in \mathbb{R}_{+}
$$

and

$$
B_{\alpha}\left[F_{s} f\right](x)=F_{s}\left[H_{\alpha} f\right](x), \quad \text { a.e } x \in \mathbb{R}_{+} .
$$

Proof. We start with the proof of (6). Let $1<p \leq 2$ and $f \in$ $L^{p}\left(\mathbb{R}_{+}\right)$. Then $F_{c} f \in L^{p^{\prime}}\left(\mathbb{R}_{+}\right)$, where $p^{\prime} \in[2, \infty)$. Applying (4) we find $B_{\alpha}\left[F_{c} f\right] \in L^{p^{\prime}}\left(\mathbb{R}_{+}\right)$. Let $a>0$ and $f_{a}(x)=f \chi_{(0, a)}(x)$, where $\chi_{(0, a)}(x)$ is the characteristic function (indicator) of an interval $(0, a)$. Then

$$
F_{c}\left(f_{a}\right)(x)=\sqrt{\frac{2}{\pi}} \int_{0}^{a} f(t) \cos x t d t .
$$

First we show that if $\alpha>1 / p^{\prime}$, then

$$
B_{\alpha}\left[F_{c} f\right](x)=\lim _{a \rightarrow \infty} B_{\alpha}\left[F_{c}\left(f_{a}\right)\right](x) \quad \text { for all } x \in \mathbb{R}_{+} .
$$

Indeed, by Hölder's and Planscherel-Titchmarsh's inequalities

$$
\left|B_{\alpha}\left[F_{c} f\right](x)-B_{\alpha}\left[F_{c}\left(f_{a}\right)\right](x)\right| \leq \frac{1}{x^{\alpha}} \int_{0}^{x}(x-y)^{\alpha-1}\left|F_{c} f(y)-F_{c}\left(f_{a}\right)(y)\right| d y
$$




$$
\leq C x^{-1 / p^{\prime}}\left\|F_{c} f-F_{c}\left(f_{a}\right)\right\|_{p^{\prime}} \rightarrow 0, \quad a \rightarrow \infty
$$

and (8) follows. Next we show that

$$
B_{\alpha}\left[F_{c}\left(f_{a}\right)\right](x)=F_{c}\left[H_{\alpha}\left(f_{a}\right)\right](x) .
$$

By the Fubini theorem and by change of variables we have

$$
\begin{aligned}
B_{\alpha}\left[F_{c}\left(f_{a}\right)\right](x) & =\frac{1}{x^{\alpha}} \int_{0}^{x}(x-y)^{\alpha-1} F_{c}\left(f_{a}\right)(y) d y \\
& =\sqrt{\frac{2}{\pi}} \frac{1}{x^{\alpha}} \int_{0}^{x}(x-y)^{\alpha-1} d y \int_{0}^{a} f(t) \cos y t d t \\
& =\sqrt{\frac{2}{\pi}} \frac{1}{x^{\alpha}} \int_{0}^{a} f(t) d t \int_{0}^{x}(x-y)^{\alpha-1} \cos y t d y=\{y t=x u\} \\
& =\sqrt{\frac{2}{\pi}} \int_{0}^{a} \frac{f(t)}{t^{\alpha}} d t \int_{0}^{t}(t-u)^{\alpha-1} \cos x u d u
\end{aligned}
$$

On the other hand

$$
\begin{aligned}
F_{c}\left[H_{\alpha}\left(f_{a}\right)\right](x) & =\sqrt{\frac{2}{\pi}} \int_{0}^{\infty} H_{\alpha}\left(f_{a}\right)(y) \cos x y d y \\
& =\sqrt{\frac{2}{\pi}} \int_{0}^{\infty} \cos x y d y \int_{y}^{\infty} \frac{(t-y)^{\alpha-1}}{t^{\alpha}} f_{a}(t) d t \\
& =\sqrt{\frac{2}{\pi}} \int_{0}^{a} \frac{f(t)}{t^{\alpha}} d t \int_{0}^{t}(t-y)^{\alpha-1} \cos x y d y
\end{aligned}
$$

and (9) follows. Now, since $f \in L^{p}\left(\mathbb{R}_{+}\right), p \in(1,2]$, then by (5) we have $H_{\alpha} f \in L^{p}\left(\mathbb{R}_{+}\right)$and $F_{c}\left[H_{\alpha} f\right](x) \in L^{p^{\prime}}\left(\mathbb{R}_{+}\right)$. We show that

$$
\lim _{a \rightarrow \infty}\left\|F_{c}\left[H_{\alpha} f\right]-F_{c}\left[H_{\alpha}\left(f_{a}\right)\right]\right\|_{p^{\prime}}=0 .
$$

Write

$$
\begin{aligned}
F_{c}\left[H_{\alpha}\left(f_{a}\right)\right](x) & =\sqrt{\frac{2}{\pi}} \int_{0}^{a} \cos x y d y \int_{y}^{a} \frac{(t-y)^{\alpha-1}}{t^{\alpha}} f(t) d t \\
& =F_{c}\left[H_{\alpha} f\right]_{a}(x)-\sqrt{\frac{2}{\pi}} \int_{0}^{a} \cos x y d y \int_{a}^{\infty} \frac{(t-y)^{\alpha-1}}{t^{\alpha}} f(t) d t .
\end{aligned}
$$


Applying Minkowskii's inequality

$$
\begin{aligned}
\left\|F_{c}\left[H_{\alpha} f\right]-F_{c}\left[H_{\alpha}\left(f_{a}\right)\right]\right\|_{p^{\prime}} \leq & \left\|F_{c}\left[H_{\alpha} f\right]-F_{c}\left[H_{\alpha} f\right]_{a}\right\|_{p^{\prime}} \\
& +\left(\int_{0}^{\infty}\left|\int_{0}^{a} \cos x y d y \int_{a}^{\infty} \frac{(t-y)^{\alpha-1}}{t^{\alpha}} f(t) d t\right|^{p \prime} d x\right)^{1 / p^{\prime}} .
\end{aligned}
$$

By Planscherel-Titchmarsh's inequality

$$
\begin{aligned}
\left\|F_{c}\left[H_{\alpha} f\right]-F_{c}\left[H_{\alpha} f\right]_{a}\right\|_{p^{\prime}} & \leq C(p)\left\|H_{\alpha} f-\left[H_{\alpha} f\right]_{a}\right\|_{p} \\
& =C(p)\left(\int_{a}^{\infty}\left|H_{\alpha} f(x)\right|^{p} d x\right)^{1 / p} \rightarrow 0, \quad a \rightarrow \infty
\end{aligned}
$$

and (10) follows if

$$
\lim _{a \rightarrow \infty} \int_{0}^{\infty}\left|\int_{0}^{a} \cos x y d y \int_{a}^{\infty} \frac{(t-y)^{\alpha-1}}{t^{\alpha}} f(t) d t\right|^{p \prime} d x=0 .
$$

By Planscherel-Titchmarsh's and Hölder's inequalities we find

$$
\begin{aligned}
\left(\int_{0}^{\infty} \mid \int_{0}^{a} \cos x y\right. & \left.\left.d y \int_{a}^{\infty} \frac{(t-y)^{\alpha-1}}{t^{\alpha}} f(t) d t\right|^{p \prime} d x\right)^{1 / p^{\prime}} \\
\leq & C\left(\int_{0}^{a}\left|\int_{a}^{\infty}\left(1-\frac{y}{t}\right)^{(\alpha-1)} \frac{f(t)}{t} d t\right|^{p} d y\right)^{1 / p} \\
\leq & C\left(\int_{0}^{a}\left(1-\frac{y}{a}\right)^{p(\alpha-1)} d y\right)^{1 / p}\left|\int_{a}^{\infty} \frac{f(t)}{t} d t\right| \\
& \left.=C_{1} a^{1 / p}\left|\int_{a}^{\infty} \frac{f(t)}{t} d t\right|^{1 / p}|f(t)|^{p} d t\right)^{1 / p} \rightarrow 0, \quad a \rightarrow \infty \\
& \leq C_{2}\left(\int_{a}^{\infty} \mid \infty\right.
\end{aligned}
$$

since $0<1-\frac{y}{a} \leq 1-\frac{y}{t} \leq 1, \quad 0 \leq y \leq a \leq t<\infty$, and (11) is proved.

Observe that (10) implies the existence of a subsequence $\left\{a_{k}\right\}, a_{k} \rightarrow \infty$ such that

$$
F_{c}\left[H_{\alpha} f\right](x)=\lim _{k \rightarrow \infty} F_{c}\left[H_{\alpha}\left(f_{a_{k}}\right)\right](x) \quad \text { a.e } x \in \mathbb{R}_{+} .
$$

Now (6) follows from this, (8) and (9). The proof of (7) is analogous.

Theorem 2. Let $f \in L^{p}\left(\mathbb{R}_{+}\right)$, where $1<p \leq 2$ and let $\alpha>1 / p^{\prime}$. Then

$$
H_{\alpha}\left[F_{c} f\right](x)=F_{c}\left[B_{\alpha} f\right](x), \quad \text { a.e. } x \in \mathbb{R}_{+}
$$


and

$$
H_{\alpha}\left[F_{s} f\right](x)=F_{s}\left[B_{\alpha} f\right](x), \quad \text { a.e. } x \in \mathbb{R}_{+} .
$$

Proof. Applying Planscherel-Titchmarsh's inequality and (4) we have $H_{\alpha}\left[F_{c} f\right](x) \in L^{p^{\prime}}\left(\mathbb{R}_{+}\right)$. For $A>x>0$ by Fubini's theorem we write

$$
\int_{x}^{A} \frac{(y-x)^{\alpha-1}}{y^{\alpha}} d y \int_{0}^{a} f(t) \cos y t d t=\int_{0}^{a} f(t) d t \int_{x}^{A} \frac{(y-x)^{\alpha-1}}{y^{\alpha}} \cos y t d y .
$$

Let us show that if $a \rightarrow+\infty$, then the equality

$$
\int_{x}^{A} \frac{(y-x)^{\alpha-1}}{y^{\alpha}} F_{c}(f)(y) d y=\sqrt{\frac{2}{\pi}} \int_{0}^{\infty} f(t) d t \int_{x}^{A} \frac{(y-x)^{\alpha-1}}{y^{\alpha}} \cos y t d y
$$

holds. Indeed, for the left hand side of (14) we have

$$
\sqrt{\frac{2}{\pi}} \int_{x}^{A} \frac{(y-x)^{\alpha-1}}{y^{\alpha}} d y \int_{0}^{a} f(t) \cos y t d t=\int_{x}^{A} \frac{(y-x)^{\alpha-1}}{y^{\alpha}} F_{c}\left(f_{a}\right)(y) d y
$$

and by Lebesgue's theorem on dominated convergence

$$
\lim _{a \rightarrow \infty} \int_{x}^{A} \frac{(y-x)^{\alpha-1}}{y^{\alpha}} F_{c}\left(f_{a}\right)(y) d y=\int_{x}^{A} \frac{(y-x)^{\alpha-1}}{y^{\alpha}} F_{c} f(y) d y .
$$

It implies that the right hand side of (14) is convergent for $a \rightarrow \infty$ and (15) holds with the first integral on the right in the Riemann sense. Now, by Hölder's inequality

$$
\begin{aligned}
\int_{x}^{\infty}\left|\frac{(y-x)^{\alpha-1}}{y^{\alpha}} F_{c} f(y) d y\right| & \leq\left\|F_{c} f\right\|_{p^{\prime}}\left(\int_{x}^{\infty} \frac{(y-x)^{(\alpha-1) p}}{y^{\alpha p}} d y\right)^{1 / p} \\
& =C\left\|F_{c} f\right\|_{p^{\prime}} x^{-\frac{1}{p^{\prime}}}<\infty
\end{aligned}
$$

Therefore, by Lebesgue's theorem on dominated convergence there exist a finite limit of the left hand side of (15)

$$
\lim _{A \rightarrow+\infty} \int_{x}^{A} \frac{(y-x)^{\alpha-1}}{y^{\alpha}} F_{c} f(y) d y=\int_{x}^{\infty} \frac{(y-x)^{\alpha-1}}{y^{\alpha}} F_{c} f(y) d y .
$$

Hence,

$$
H_{\alpha}\left[F_{c} f\right](x)=\sqrt{\frac{2}{\pi}} \int_{0}^{\infty} f(t) d t \int_{x}^{\infty} \frac{(y-x)^{\alpha-1}}{y^{\alpha}} \cos y t d y
$$


for all $x \in \mathbb{R}_{+}$with both the integrals on the right in the Riemann sense. To justify (16) with both the integrals on the right in the Lebesgue sense, we consider the function from the right hand side of (15)

$$
h_{x, A}(t):=\int_{x}^{A} \frac{(y-x)^{\alpha-1}}{y^{\alpha}} \cos y t d y,
$$

when $t \rightarrow+0$ and $t \rightarrow+\infty$. If $t \rightarrow+0$, then

$$
\begin{aligned}
h_{x, A}(t) & =\int_{x t}^{A t} \frac{(z-x t)^{\alpha-1}}{z^{\alpha}} \cos z d z \\
& =\int_{u}^{A u / x} \frac{(z-u)^{\alpha-1}}{z^{\alpha}} \cos z d z \\
& =\int_{0}^{(A / x-1) u} \frac{s^{\alpha-1}}{(s+u)^{\alpha}} \cos (s+u) d s:=W(u)
\end{aligned}
$$

To estimate $W(u)$, we shall use the asymptotic formulae from [2, Chapter 1 , Section 4]. Let $\theta \in \mathbb{R}, \beta>0, \varphi(t) \in C[0, a]$ and let $\theta+\beta=N$, where $N$ is a non-negative integer. Then

$$
\begin{aligned}
& \int_{0}^{a} t^{\beta-1}(t+\varepsilon)^{\theta} \varphi(t, \varepsilon) d t \\
& \left.\sim \sum_{n \geq \max [0,-N]}^{N}\left(\begin{array}{c}
n+N \\
\theta
\end{array}\right) \frac{\partial^{n} \varphi(t, \varepsilon)}{\partial t^{n}}\right|_{t=0} \varepsilon^{n+N} \ln (1 / \varepsilon)+\sum_{n=0}^{\infty} b_{n} \varepsilon^{n}
\end{aligned}
$$

for $\varepsilon \rightarrow 0$, if $\left.\varepsilon \in S_{\delta}:=\{0<|\varepsilon| \leq r,|\arg \varepsilon| \leq \pi-\delta]\right\} \subset \mathbb{C}, b_{n}$ are constants and $\varphi(t, \varepsilon) \in C^{\infty}([0, a] \times[\varepsilon:|\varepsilon|<r])$.

If we take $\varphi(s, u):=\cos (s+u), \beta:=\alpha, \theta:=-\alpha$, so that $\theta+\beta=N=0$, then by (17), we find

$$
W(u) \sim \cos u \ln (1 / u), \quad u \rightarrow+0 .
$$

Hence,

$$
h_{x, A}(t)=O(\ln (1 / x t)), \quad t \rightarrow+0 .
$$

For the case $t \rightarrow+\infty$ we write

$$
\begin{aligned}
h_{x, A}(t) & =\int_{x}^{A} \frac{(y-x)^{\alpha-1}}{y^{\alpha}} \cos y t d y=\int_{0}^{A-x} \frac{y^{\alpha-1}}{(y+x)^{\alpha}} \cos t(y+x) d y \\
& =\Re e^{i x t} \int_{0}^{A-x} g(y) d\left(-\int_{y}^{\infty} u^{\alpha-1} e^{i u t} d u\right)=: \Re e^{i x t} \Phi_{\alpha}(t),
\end{aligned}
$$


where $g(y)=(y+x)^{-\alpha}$. Integrating by parts, we obtain

$$
\begin{aligned}
\Phi_{\alpha}(t)=g(0) & \int_{0}^{\infty} u^{\alpha-1} e^{i u t} d u-g(A-x) \int_{A-x}^{\infty} u^{\alpha-1} e^{i u t} d u \\
& +\int_{0}^{A-x}\left(\int_{y}^{\infty} u^{\alpha-1} e^{i u t} d u\right) g^{\prime}(y) d y .
\end{aligned}
$$

Put $u-y=\rho e^{i \frac{\pi}{2}}$ and $u=r e^{i \theta}$, then $\rho \leq r, 0 \leq \theta \leq \frac{\pi}{2}$. If $|u| \geq y$, then $|u|^{\alpha-1} \leq y^{\alpha-1}$, so that

$$
\left|\int_{y}^{\infty} u^{\alpha-1} e^{i u t} d u\right| \leq y^{\alpha-1} \int_{0}^{\infty} e^{-t \rho} d \rho=\Gamma(1) y^{\alpha-1} t^{-1} .
$$

Hence,

$$
\int_{0}^{A-x}\left(\int_{y}^{\infty} u^{\alpha-1} e^{i u t} d u\right) g^{\prime}(y) d y=O\left(t^{-1}\right) .
$$

Analogously,

$$
\int_{A-x}^{\infty} u^{\alpha-1} e^{i u t} d u=O\left(t^{-1}\right)
$$

and also it is known that

$$
\int_{0}^{\infty} u^{\alpha-1} e^{i u t} d u=e^{-\pi i \alpha / 2} t^{-\alpha} \Gamma(\alpha)
$$

Therefore,

$$
h_{x, A}(t)=O\left(t^{-\alpha}\right), \quad t \rightarrow+\infty .
$$

Thus, it follows from (18) and (19) that there exist a function

$$
G(t)=|f(t)|\left\{\chi_{[0,1 / 2]}(t)|\ln (1 / x t)|+\chi_{[1 / 2, \infty)}(t) t^{-\alpha}\right\} \in L^{1}(0, \infty)
$$

such that

$$
\left|f(t) h_{x, A}(t)\right| \leq G(t)
$$

By Lebesgue's theorem on dominated convergence

$$
\int_{0}^{\infty} f(t) d t \int_{x}^{\infty} \frac{(y-x)^{\alpha-1}}{y^{\alpha}} \cos y t d y=\lim _{A \rightarrow \infty} \int_{0}^{\infty} f(t) h_{x, A}(t) d t .
$$

It implies

$$
H_{\alpha}\left[F_{c} f\right](x)=\sqrt{\frac{2}{\pi}} \int_{0}^{\infty} f(t) h_{x, \infty}(t) d t .
$$


By change of variables we find

$$
\begin{aligned}
h_{x, \infty}(t) & =\int_{x}^{\infty} \frac{(y-x)^{\alpha-1}}{y^{\alpha}} \cos y t d y=\{y t \rightarrow v\} \\
& =\int_{x t}^{\infty} \frac{(v-x t)^{\alpha-1}}{v^{\alpha}} \cos v d v=\{v \rightarrow x y\} \\
& =\int_{t}^{\infty} \frac{(y-t)^{\alpha-1}}{y^{\alpha}} \cos x y d y=h_{t, \infty}(x) .
\end{aligned}
$$

It follows from this and (20) that

$$
H_{\alpha}\left[F_{c} f\right](x)=\sqrt{\frac{2}{\pi}} \int_{0}^{\infty} f(t) d t \int_{t}^{\infty} \frac{(y-t)^{\alpha-1}}{y^{\alpha}} \cos x y d y .
$$

Now we show that

$$
H_{\alpha}\left[F_{c} f\right](x)=\lim _{a \rightarrow \infty} \sqrt{\frac{2}{\pi}} \int_{0}^{a} f(t) d t \int_{t}^{a} \frac{(y-t)^{\alpha-1}}{y^{\alpha}} \cos y x d y .
$$

We have

$$
\begin{aligned}
& \left|\int_{a}^{\infty} \frac{(y-t)^{\alpha-1}}{y^{\alpha}} \cos x y d y\right| \\
& \quad=\frac{1}{x}\left|\frac{(y-t)^{\alpha-1}}{y^{\alpha}} \sin x y\right|_{a}^{\infty}-\int_{a}^{\infty} \sin x y d\left(\frac{(y-t)^{\alpha-1}}{y^{\alpha}}\right) \mid \\
& \leq \frac{(a-t)^{\alpha-1}}{a^{\alpha} x}+\frac{1}{x} \int_{a}^{\infty}\left|d\left(\frac{(y-t)^{\alpha-1}}{y^{\alpha}}\right)\right| \leq \frac{2(a-t)^{\alpha-1}}{a^{\alpha} x}
\end{aligned}
$$

then

$$
\begin{gathered}
\left|\int_{0}^{a} f(t) d t \int_{t}^{\infty} \frac{(y-t)^{\alpha-1}}{y^{\alpha}} \cos y x d y-\int_{0}^{a} f(t) d t \int_{t}^{a} \frac{(y-t)^{\alpha-1}}{y^{\alpha}} \cos y x d y\right| \\
\quad=\left|\int_{0}^{a} f(t) d t \int_{a}^{\infty} \frac{(y-t)^{\alpha-1}}{y^{\alpha}} \cos y x d y\right| \leq \int_{0}^{a} \frac{2(a-t)^{\alpha-1}}{a^{\alpha} x}|f(t)| d t
\end{gathered}
$$

and by Hölder's inequality

$$
\lim _{a \rightarrow \infty} \int_{0}^{a} \frac{2(a-t)^{\alpha-1}}{a^{\alpha} x}|f(t)| d t \leq \lim _{a \rightarrow \infty} 2 C(\alpha, p) \frac{a^{\alpha-1 / p}}{a^{\alpha} x}\|f\|_{L^{p}}=0 .
$$

Again applying Hölder's inequality

$$
\left|\int_{0}^{\infty} f(t) d t \int_{t}^{\infty} \frac{(y-t)^{\alpha-1}}{y^{\alpha}} \cos y x d y-\int_{0}^{a} f(t) d t \int_{t}^{\infty} \frac{(y-t)^{\alpha-1}}{y^{\alpha}} \cos y x d y\right|
$$


$=\left|\int_{a}^{\infty} f(t) h_{x, A}(t) d t\right| \leq C\left(\int_{a}^{\infty}|f|^{p} d t\right)^{1 / p}\left(\int_{a}^{\infty} t^{-\alpha p^{\prime}} d t\right)^{1 / p^{\prime}} \rightarrow 0, a \rightarrow \infty$ and (21) follows. Changing the order of integrals on the right hand side of (21), we find

$$
H_{\alpha}\left[F_{c} f\right](x)=\lim _{a \rightarrow \infty} F_{c}\left[B_{\alpha} f\right]_{a}(x)
$$

Since

$$
\lim _{a \rightarrow \infty}\left\|F_{c}\left[B_{\alpha} f\right]-F_{c}\left[B_{\alpha} f\right]_{a}\right\|_{p^{\prime}}=0,
$$

then there exist a sequence $a_{k}$ such that $a_{k} \rightarrow \infty, k \rightarrow \infty$ and

$$
F_{c}\left[B_{\alpha} f\right]=\lim _{k \rightarrow \infty} F_{c}\left[B_{\alpha} f\right]_{a_{k}}(x) \text {, a.e. } x \in \mathbb{R}_{+}
$$

and (12) follows from (22), (23) and (24). The proof of (13) is analogous.

For the case $p=1$ we have the following analog of Theorems 1 and 3 .

Theorem 3. If $f \in L^{1}\left(\mathbb{R}_{+}\right)$and $\alpha>0$, then for all $x \in \mathbb{R}_{+}$the equalities

$$
B_{\alpha}\left[F_{c} f\right](x)=F_{c}\left[H_{\alpha} f\right](x), B_{\alpha}\left[F_{s} f\right](x)=F_{s}\left[H_{\alpha} f\right](x)
$$

hold.

Proof. By Fubini theorem we write

$$
\begin{aligned}
B_{\alpha}\left[F_{c} f\right](x) & =\frac{1}{x^{\alpha}} \int_{0}^{x}(x-y)^{\alpha-1} F_{c} f(y) d y \\
& =\sqrt{\frac{2}{\pi}} \frac{1}{x^{\alpha}} \int_{0}^{x}(x-y)^{\alpha-1}\left\{\int_{0}^{\infty} f(t) \cos y t d t\right\} d y . \\
& =\sqrt{\frac{2}{\pi}} \frac{1}{x^{\alpha}} \int_{0}^{\infty} f(t)\left\{\int_{0}^{x}(x-y)^{\alpha-1} \cos y t d y\right\} d t
\end{aligned}
$$

Changing $t y=x u$, we have $x-y=\frac{x t-x u}{t}, d y=\frac{x}{t} d u$ and

$$
B_{\alpha}\left[F_{c} f\right](x)=\sqrt{\frac{2}{\pi}} \int_{0}^{\infty} \frac{f(t)}{t^{\alpha}}\left\{\int_{0}^{t}(t-u)^{\alpha-1} \cos x u d u\right\} d t .
$$


On the other hand again by Fubini's theorem

$$
\begin{aligned}
F_{c}\left[H_{\alpha} f\right](x) & =\sqrt{\frac{2}{\pi}} \int_{0}^{\infty} H_{\alpha} f(y) \cos x y d y \\
& =\sqrt{\frac{2}{\pi}} \int_{0}^{\infty} \cos x y\left\{\int_{y}^{\infty} \frac{(t-y)^{\alpha-1}}{t^{\alpha}} f(t) d t\right\} d y \\
& =\sqrt{\frac{2}{\pi}} \int_{0}^{\infty} \frac{f(t)}{t^{\alpha}}\left\{\int_{0}^{t}(t-y)^{\alpha-1} \cos x y d y\right\} d t
\end{aligned}
$$

and for all $x>0$ the equality

$$
B_{\alpha}\left[F_{c} f\right](x)=F_{c}\left[H_{\alpha} f\right](x)
$$

follows. If $x=0$ then

$$
F_{c}\left[H_{\alpha} f\right](0)=\sqrt{\frac{2}{\pi}} \frac{1}{\alpha} \int_{0}^{\infty} f(t) d t=\frac{1}{\alpha} F_{c} f(0)
$$

and for a continuous $f$ at $x=0$ we have

$$
B_{\alpha} f(0):=\lim _{x \rightarrow 0} B_{\alpha} f(x)=\lim _{x \rightarrow 0} \frac{1}{x^{\alpha}} \int_{0}^{x}(x-t)^{\alpha-1} f(t) d t=\frac{1}{\alpha} f(0) .
$$

Therefore, if $f \in L^{1}\left(\mathbb{R}_{+}\right)$, then $F_{c} f(x)$ is continuous and

$$
B_{\alpha}\left[F_{c} f\right](0):=\lim _{x \rightarrow 0} B_{\alpha}\left[F_{c} f\right](x)=\frac{1}{\alpha} F_{c} f(0) .
$$

Now we extend the operators $B_{\alpha}$ and $H_{\alpha}$ on $\mathbb{R}$ as follows.

$$
B_{\alpha} f(x):=\left\{\begin{array}{cc}
\frac{1}{x^{\alpha}} \int_{0}^{x}(x-t)^{\alpha-1} f(t) d t, & x>0 \\
\frac{1}{|x|^{\alpha}} \int_{x}^{0}(|x|-|t|)^{\alpha-1} f(t) d t, & x<0
\end{array}\right.
$$

and

$$
H_{\alpha} f(x):=\left\{\begin{array}{cc}
\int_{x}^{\infty} \frac{(t-x)^{\alpha-1}}{t^{\alpha}} f(t) d t, & x>0 \\
\int_{-\infty}^{x} \frac{(|t|-|x|)^{\alpha-1}}{|t|^{\alpha}} f(t) d t, & x<0 .
\end{array}\right.
$$

It is easy to see, that for even or odd functions $f(x)$, the images $B_{\alpha} f(x)$ and $H_{\alpha} f(x)$ are even or odd too. As a consequence of Theorems 1 and 2, we obtain the following result. 
Theorem 4. If $1<p \leq 2$ and $\alpha>1 / p^{\prime}$, then

$$
B_{\alpha}[F f](x)=F\left[H_{\alpha} f\right](x), \quad \text { a.e } x \in \mathbb{R}
$$

and

$$
H_{\alpha}[F f](x)=F\left[B_{\alpha} f\right](x), \quad \text { a.e } x \in \mathbb{R}
$$

for any $f \in L^{p}(\mathbb{R})$.

\section{References}

[1] R. Bellman, A note on a theorem of Hardy on Fourier constants, Bull. Amer. Math Soc., 50 (1944), 741-744.

[2] M.V. Fedoryuk, The saddle-point method (Russian), Izdat. Nauka, Moscow, 1977.

[3] B.I. Golubov, On a theorem of Bellman on Fourier coefficients, Russian Acad. Sci. Sb. Math, 83 (1995), 321-330.

[4] G.H. Hardy, J.E. Littlewood and G. Pólya, Inequalities, Cambridge Univ. Press, 1952.

[5] F. Moricz, The harmonic Cesaro and Copson operators on the spaces $L^{p}(\mathbb{R}), 1 \leq p \leq 2$, Studia Math., 149 (2002), 267-279.

[6] A.C. Titchmarsh, Introduction to the Theory of Fourier's Integrals, Oxford Univ. Press, 1937.

Department of Mathematical Analysis and Function Theory

Peoples Friendship University of Russia

Miklukho Maklai 6

Moscow 117198

Russia

(E-mail : ptdung2004@mail.ru)

(Received : February 2009) 


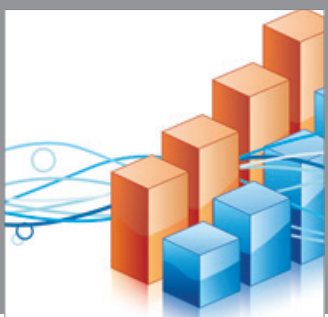

Advances in

Operations Research

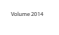

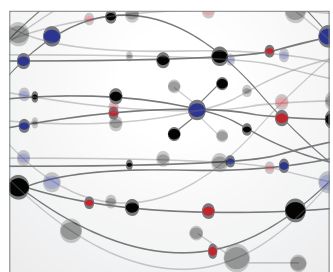

\section{The Scientific} World Journal
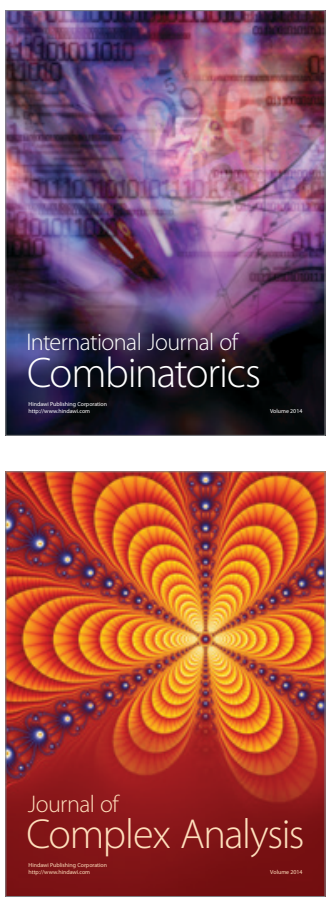

International Journal of

Mathematics and

Mathematical

Sciences
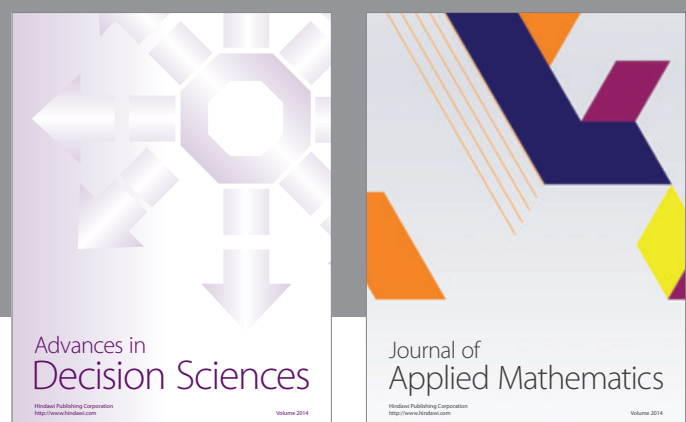

Journal of

Applied Mathematics
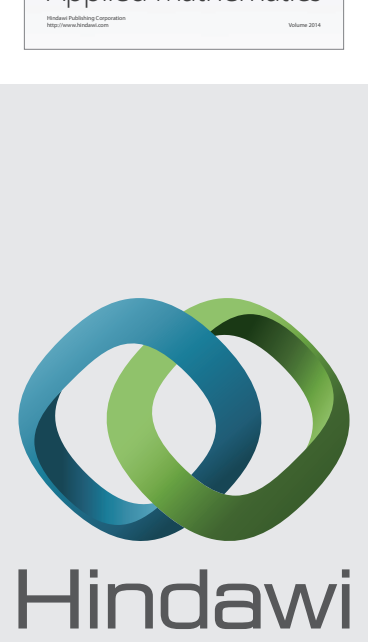

Submit your manuscripts at http://www.hindawi.com
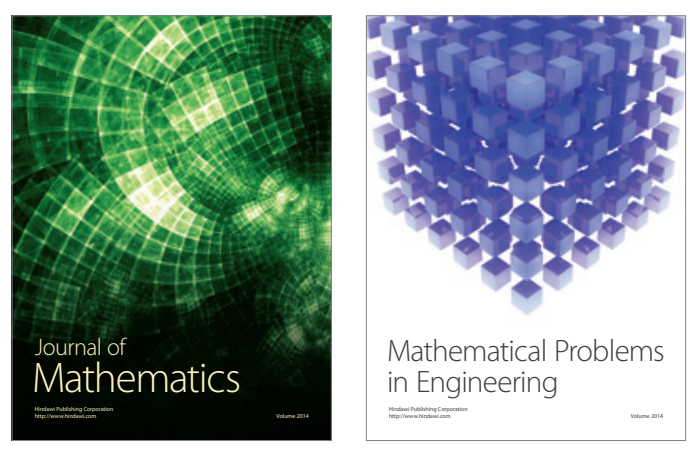

Mathematical Problems in Engineering
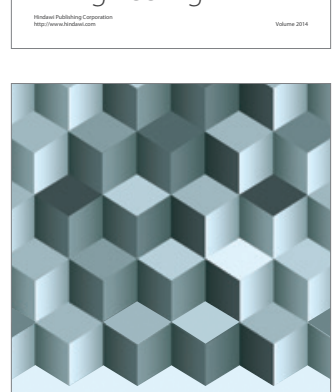

Journal of

Function Spaces
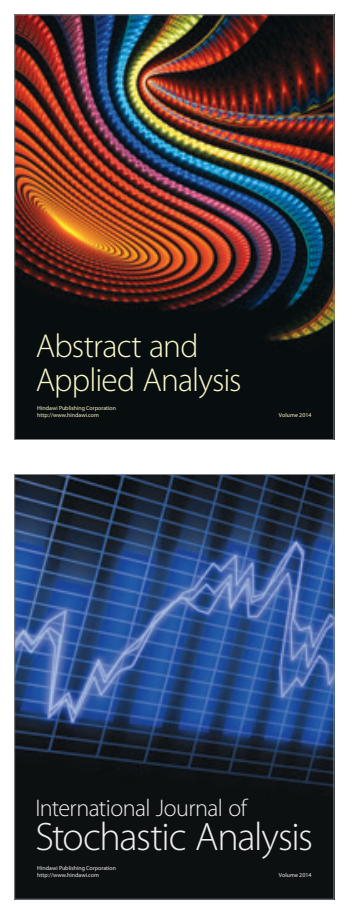

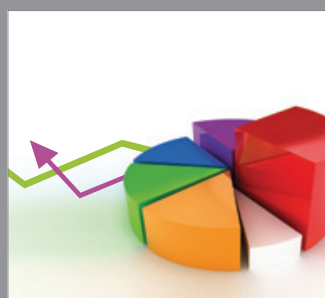

ournal of

Probability and Statistics

Promensencen
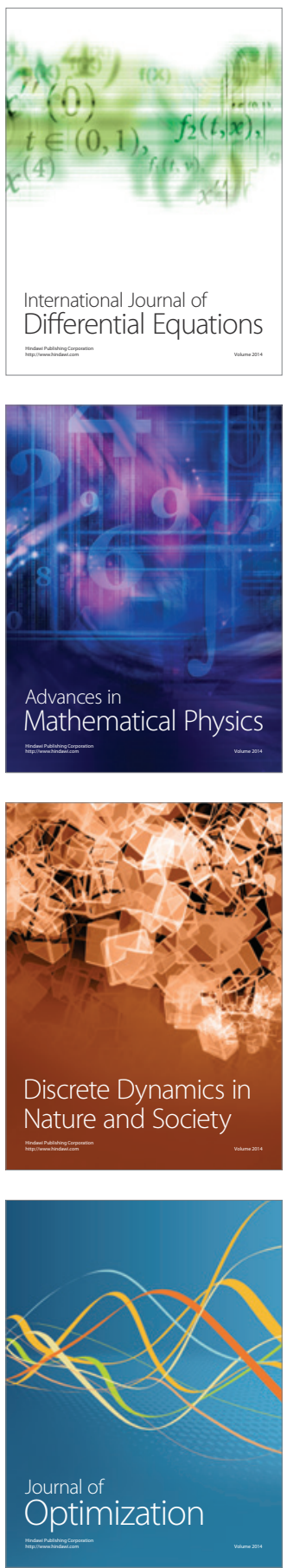